\title{
NORMED LINEAR SPACES OF CONTINUOUS FUNCTIONS
}

\section{S. B. MYERS}

1. Introduction. In addition to its well known role in analysis, based on measure theory and integration, the study of the Banach space $B(X)$ of real bounded continuous functions on a topological space $X$ seems to be motivated by two major objectives.

The first of these is the general question as to relations between the topological properties of $X$ and the properties (algebraic, topological, metric) of $B(X)$ and its linear subspaces. The impetus to the study of this question has been given by various results which show that, under certain natural restrictions on $X$, the topological structure of $X$ is completely determined by the structure of $B(X)[3 ; 16 ; 7],{ }^{1}$ and even by the structure of a certain type of subspace of $B(X)$ [14]. Beyond these foundational theorems, the results are as yet meager and exploratory. It would be exciting (but surprising) if some natural metric property of $B(X)$ were to lead to the unearthing of a new topological concept or theorem about $X$.

The second goal is to obtain information about the structure and classification of real Banach spaces. The hope in this direction is based on the fact that every Banach space is (equivalent to) a linear subspace of $B(X)$ [1] for some compact (that is, bicompact Hausdorff) $X$. Properties have been found which characterize the spaces $B(X)$ among all Banach spaces $[6 ; 2 ; 14]$, and more generally, properties which characterize those Banach spaces which determine the topological structure of some compact or completely regular $X$ $[14 ; 15]$. These properties are defined in terms of concepts which are meaningful in all Banach spaces; in particular, no lattice [10] or ring $[8 ; 9 ; 11]$ structure is presupposed.

I propose here to outline and supplement some recent results along the above two lines, using methods developed in [14]. In one or two instances details of proofs are given; these are brief and have not previously appeared in print.

2. The mapping $C(X)$. Let $X$ be a completely regular topological space. The set of all real-valued, bounded continuous functions on $X$, with the usual laws of addition and multiplication by real num-

An address delivered before the Chicago Meeting of the Society, February 26, 1949 , by invitation of the Committee to Select Hour Speakers for Western Sectional Meetings; received by the editors April 23, 1949.

${ }^{1}$ Numbers in brackets refer to the bibliography at the end of the paper. 
bers, is made into a Banach space $B(X)$ over the reals by defining $\|b\|=\sup _{x \in X}|b(x)|$. Let $B$ be a closed linear subspace of $B(X)$. Consider the following possibilities:

(I) $B$ is separating over $X$; that is, given $x_{1}, x_{2} \in X, x_{1} \neq x_{2}$, there exists a $b \in B$ such that $b\left(x_{1}\right) \neq b\left(x_{2}\right)$.

(II) Given $x \in X$, and a closed set $X_{1} \subset X$ not containing $x$, there exists a finite set $b_{1}, \cdots, b_{n} \in B$ and a $\delta>0$, such that, for each $x_{1} \in X_{1},\left|b_{i}(x)-b_{i}\left(x_{1}\right)\right| \geqq \delta$ for at least one value of $i$.

(III) $B$ is completely regular over $X$; that is, given $x \in X$ and a closed set $Y \subset X$ not containing $x$, there exists a $b \in B$ such that $b(x)=\|b\|$, $\sup _{y \in Y}|b(y)|<\|b\|$.

It is easy to see that (III) $\rightarrow$ (II) $\rightarrow$ (I). If $X$ is compact, (I) $=(\mathrm{II})$.

Now let $B_{W}^{*}$ be the conjugate space to $B$, provided with the weak-* topology, let $E_{W}^{*}$ be its compact $[1 ; 4]$ solid unit sphere. For each $x \in X$, let $x^{*}$ be the point in $E_{W}^{*}$ defined by $x^{*}(b)=b(x)$ for all $b \in B$. The mapping $C$ of $X$ into $E_{W}^{*}$ defined by $C(x)=x^{*}$ for all $x \in X$ is always continuous, $C(X)$ is a total subset of $B_{W}^{*}$, and for each $b \in B$, $\|b\|=\sup _{f \in C(X)}|f(b)| . C$ is one-to-one if and only if $B$ satisfies (I). It is a homeomorphism if and only if $B$ satisfies (II).

Given any Banach space $B$, there always exists a compact $X$ such that $B$ is equivalent to a closed linear subspace of $B(X)$ satisfying (II); for example, $X$ can be taken as $E_{W}^{*}$ in $B_{W}^{*}$. However, the class of Banach spaces satisfying (III) with some completely regular $X$ is, as we shall see, a proper subclass of all Banach spaces, and itself contains as a proper subclass the class of Banach spaces satisfying (III) with some compact $X$. We shall characterize both of these classes. Also, we shall see that a given Banach space $B$ satisfies (III) with at most one compact $X$.

3. Spaces completely regular over $X[14 ; 15]$. Let $B$ be an arbitrary Banach space. Each $b \in B$ is contained in a $T$-set-that is, a subset of $B$ maximal with respect to the property that for each of its finite subsets $\left(b_{1}, \cdots, b_{n}\right),\left\|\sum b_{i}\right\|=\sum\left\|b_{i}\right\|$. The intersections of $T$-sets with $S$, the surface of the unit sphere in $B$, are the maximal convex subsets of $S$ used by Eilenberg [7]. For each $T$-set $T$ we define a functional $F_{T}$ by the formula $F_{T}(b)=\inf _{t \in T}(\|b+t\|-\|t\|)$. Each $F_{T}$ is continuous over $B$ and is convex, and $\left|F_{T}(b)\right| \leqq\|b\|$, with $F_{T}(t)=\|t\|$ for $t \in T . F_{T}$ is linear over $B$ if and only if $F_{T}(b)=$ $-F_{T}(-b)$ for all $b \in B$. Also, $F_{T}$ is linear if and only if there exists a unique $f \in S_{W}^{*}$ such that $f(t)=\|t\|$ for all $t \in T$, and in this case $f=F_{T}$ over $B$; this follows from a theorem on unique norm-preserving extensions of a linear functional, which is stated and proved in $§ 8$. Thus 
if $F_{T}$ is linear, given any point $f \in E_{W}^{*}$ different from $F_{T}$ there is a $b \in B$ such that the continuous function over $B_{W}^{*}$ defined by $b$ according to $b(F)=F(b)$ for all $F \in B_{W}^{*}$ has the properties $b\left(F_{T}\right)=\|b\|, b(f)$ $<\|b\|$; further, $b(f)=-\|b\|$ only if $f=-F_{T}$. If all the functionals $F_{T}$ are linear, they form a total subset $M$ of $B_{W}^{*}$, contained in $S_{W}^{*}$, such that for each $b \in B,\|b\|=\sup _{f \in M}|f(b)| ; B$ is equivalent to a closed linear subspace of $B(M)$. If furthermore $M$ is the union of two disjoint closed antipodal subsets $\Omega$ and $-\Omega$, then $B$ is equivalent to a closed linear subspace of $B(\Omega)$ completely regular over $\Omega$. If $M$ is closed in $E_{W}^{*}, \Omega$ is compact.

Conversely, let $X$ be a compact space, and let $B$ be a closed linear subspace of $B(X)$. For each $T$-set $T$ in $B$ there is an $x \in X$ such that either $T=\{b \in B \mid b(x)=\|b\|\}$ or $T=\{b \in B \mid b(x)=-\|b\|\}$. Now assume $B$ is completely regular over $X$; then the $x$ of the previous statement is unique, and also every set of the form $\{b \in B \mid b(x)=\|b\|\}$ or $\{b \in B \mid b(x)=-\|b\|\}$ is a $T$-set. We denote the $T$-sets corresponding to $x$ by $T_{x}$ and $-T_{x}$. A fundamental fact is that for every $b \in B$ and $x \in X$

$$
b(x)=\inf _{t \in T_{x}}(\|b+t\|-\|t\|)=F_{T_{x}}(b) .
$$

It follows that each $F_{T}$ is linear. The set of linear functionals $F_{T}$, considered as points in $S_{W}^{*}$, is the union of two disjoint closed antipodal subsets, one of which is $C(X)$ and the other $-C(X)$.

Thus we have the following set of necessary and sufficient conditions that there exists a compact $X$ such that a given Banach space $B$ is equivalent to a linear subspace of $B(X)$ completely regular over $X$ :

(1) All the functionals $F_{T}$ are linear.

$\left(\mathrm{A}_{1}\right)$ (2) They form a closed subset $M$ of $E_{W}^{*}$.

(3) $M$ is the union of 2 disjoint antipodal closed subsets $\Omega$ and $-\Omega$.

Next, to study the case of a non-compact $X$, it is natural to attempt to compactify $X$ so as to reduce the problem to the compact case. Let $B$ be a closed linear subspace of $B(X)$ satisfying (II). We define a compactification of $X$ with respect to $B$ to be a compact space $\bar{X}$ in which $X$ is dense and such that all the functions in $B$ are extendable so as to be continuous over $\bar{X}$ and form a set which is separating over $\bar{X}$. Then there is a unique compactification of $X$ with respect to $B$; it is simply the closure of $C(X)$ in $E_{W}^{*}$. Unfortunately, if $B$ is completely regular over $X$, it will not in general be completely regular over $\bar{X}$ (see $\$ 5$ for an example), so that we cannot completely reduce the problem to the compact case. Of course, if 
$B$ is the whole of $B(X), \bar{X}$ is the Tychonoff-Cech-Stone $[17 ; 5 ; 16]$ compactification of $X$ and $B$ is c.r. over $\bar{X}$.

Let $B$ be a closed linear subspace of $B(X)$ completely regular over $X$. The sets $T_{x}=\{b \in B \mid b(x)=\|b\|\}$ and the sets $-T_{x}$ are still $T$-sets, but there may be "free" $T$-sets not of such form. However, considering $B$ as a subspace of $B(\bar{X})$, each $T$-set is of the form $T_{\bar{x}}$ or $-T_{\bar{x}}$ for some $\bar{x} \in \bar{X}$. For each $x \in X, b(x)$ can be evaluated by $\inf _{t \in T_{x}}(\|b+t\|-\|t\|)$, so that the functionals $F_{T_{x}}$ and $-F_{T_{x}}$ are linear. The set $M \subset S_{W}^{*}$ of all those functionals $F_{T}$ which are linear is not in general closed in $E_{W}^{*}$; but it is the union of two disjoint closed antipodal subsets $\Omega$ and $-\Omega$, which respectively contain as dense subsets $C(X)$ and $-C(X) . \Omega$ is homeomorphic to a dense subset of $\bar{X}$, which is the whole of $\bar{X}$ if $B$ is completely regular over $\bar{X}$.

Conversely, let $B$ be any Banach space. If the set $M \subset S_{W}^{*}$ of those functionals $F_{T}$ which are linear is large enough to have the property that, for each $b \in B,\|b\|=\sup _{f \in M}|f(b)|$, and if $M$ is the union of two disjoint closed antipodal subsets $\Omega$ and $-\Omega$, then $B$ is equivalent to a closed linear subspace of $B(\Omega)$ completely regular over $\Omega$.

Thus a given Banach space $B$ is equivalent for some completely regular $X$ to a closed linear subspace of $B(X)$ completely regular over $X$ if and only if the set $M \subset S_{W}^{*}$ of those functionals $F_{T}$ which are linear satisfies the following conditions:

(1) for each $b \in B,\|b\|=\sup _{f \in M}|f(b)|$.

$\left(\mathrm{A}_{2}\right) \quad(2) \quad M$ is the union of two disjoint antipodal closed (in $M$ ) subsets.

4. The whole space $B(X)$. Necessary and sufficient conditions that $B$ be equivalent to the whole of $B(X)$ for some compact $X$ can also be phrased in terms of the functionals $F_{T}$ [14]. They are as follows.

(1) All $F_{T}$ are linear.

(2) There exists a unit element $e \in B$; that is, an element $e$ of norm 1 such that $\left|F_{T}(e)\right|=1$ for all $F_{T}$.

(3) For each $b_{1} \in B$, there exists a $b_{2} \in B$ such that $F_{T}\left(b_{2}\right)$ $=\left|F_{T}\left(b_{1}\right)\right|$ for all $F_{T}$ for which $F_{T}(e)=1$.

The sufficiency of these conditions is based on Kakutani's [10] analogue for Banach lattices of the Stone-Weierstrass approximation theorem [16]; namely, if a closed linear sublattice of the lattice $M(X)$ of all continuous functions on a compact $X$ contains the constant functions and is separating over $X$, then it is the whole of $M(X)$. Here the set of $F_{T}$ such that $F_{T}(e)=1$, with the weak-* topology, serves as $X$. 
Condition (1) can be replaced by

(1)' The linear extension of each $T$-set in $B$ is the whole of $B$. Condition (1)' is stronger than (1) for the general Banach space $B$. In fact, (1)' does not usually hold even if $B$ is equivalent to a subspace of $B(X)$ completely regular over $X$, although, as we have seen, (1) does hold.

Condition (2) can be replaced by

(2)' There exists an $e \in B$ such that for each $b \in B$ either $\|b+e\|$ $=\|b\|+1$ or $\|b-e\|=\|b\|+1$.

Condition (3) can be replaced by

$(3)^{\prime}$ For each $b_{1}, b_{2} \in B$ there exists a $b_{3} \in B$ such that $F_{T}\left(b_{3}\right)$ $=F_{T}\left(b_{1}\right) \cdot F_{T}\left(b_{2}\right)$ for all $F_{T}$ such that $F_{T}(e)=1$.

Obviously (3)' enables us to use the Stone-Weierstrass approximation theorem.

Clarkson's solution [6] of the problem of characterizing $B(X)$ consists essentially of condition $(2)^{\prime}$, together with the demand that the half cone in $B$ with vertex $e$ and directrix $E$ has the property that the intersection of any two of its translates is itself a translate. This latter condition is sufficient to define a lattice structure on $B$ and to show that it is an $(M)$-space in the sense of Kakutani.

The solutions given by Arens and Kelley [2] to the same problem do not appeal directly to any lattice theorem to guarantee that $B$ is the whole of $B(X)$. One of their sets of conditions consists essentially of $(1)^{\prime}$ and $(2)^{\prime}$ plus a condition which insures that there exists a $b \in B$ which "separates" any given pair of disjoint closed sets in $X$; this turns out to guarantee that $B$ is the whole of $B(X)$. Their other set of conditions is stated in terms of geometrical properties of $E_{W}^{*}$, and involves a proof that the set $C(X)$ in $E_{W}^{*}(X)$ is simply the set of extreme points in $E_{W}^{*}(X)$ (see also [13]).

5. Examples. Here are a few examples to show the various possibilities when $B$ is completely regular over a non-compact $X$, and the more elegant results when $B$ is completely regular over a compact $X$.

Let $X$ be the open interval $\pi / 4<x<3 \pi / 4$ of the $x$-axis, and let $B$ be the two-dimensional Banach space consisting of all the functions $b(x)=c \sin x+d \sin 2 x$ as $c, d$ range over the reals, with norm defined as $\sup _{x \in X}|b(x)|$. The compactification of $X$ with respect to $B$ is the closed interval $\bar{X}=[\pi / 4 \leqq x \leqq 3 \pi / 4] . B$ is completely regular over $X$, but not over $\bar{X}$; for every $b \in B$ such that $b(\pi / 4)$ $=\|b\|$ has the property $b(3 \pi / 4)=-\|b\|$. The sets $\{b \in B \mid b(\pi / 4)$ $=\|b\|\}$ and $\{b \in B \mid b(\pi / 4)=-\|b\|\}$ are $T$-sets, but the corresponding functionals $F_{T}$ are not linear. The set $M$ of those functionals $F_{T}$ which 
are linear forms a subset of $S_{W}^{*}$ consisting of two disjoint homeomorphic images of $X$ whose weak-* closures are disjoint sets in $S_{W}^{*}$ homeomorphic to $\bar{X}$.

Now let $X$ be the interval $2^{1 / 2}-1<x \leqq 1$, and let $B$ be the set of functions $b(x)=c x^{2}+d x$ with $\|b\|=\sup _{x \in X}|b(x)|$. Here $\bar{X}=\left[2^{1 / 2}-1 \leqq x \leqq 1\right]$. $b\left(2^{1 / 2}-1\right)=\|b\|$ if and only if $d=-2 c\left(2^{1 / 2}-1\right), c<0$, in which case $b(1)=-\|b\| . B$ is completely regular over $X$ but not over $\bar{X}$. However, whenever $d<-2 c\left(2^{1 / 2}-1\right)$ and $c<0$, we have $b(1)=-\|b\|$ and $-\|b\|<b\left(2^{1 / 2}-1\right)<\|b\|$. Hence $\left\{b \in B \mid b\left(2^{1 / 2}-1\right)=\|b\|\right\}$ is not a $T$-set, and the functionals $F_{T}$ are all linear; as in the previous example they form a subset $M$ of $S_{W}^{*}$ consisting of two disjoint homeomorphs of $X$, whose weak closures are disjoint sets in $S_{W}^{*}$ homeomorphic to $\bar{X}$.

If $X=[0<x<1]$, let $B$ be the set of all continuous functions on $X$ such that $\lim _{x \rightarrow 0} b(x)=\lim _{x \rightarrow 1} b(X)=0$. Here $\bar{X}$ is homeomorphic to a circle. $B$ is completely regular over $X$, but not over $\bar{X}$. The functionals $F_{T}$ are all linear and form a subset of $S_{W}^{*}$ whose closure in $E_{W}^{*}$ is obtained by adjoining the origin in $B_{W}^{*}$.

The set of functions $a x^{2}+b x+c$ is completely regular over $X$ $=[0<x<1]$, and also over $\bar{X}=[0 \leqq x \leqq 1]$. The functionals $F_{T}$ are all linear, and form a subset of $S_{W}^{*}$ closed in $E_{W}^{*}$ consisting of two disjoint subsets each homeomorphic to $\bar{X}$.

6. Some classes of Banach spaces. Let

$\beta=$ class of all Banach spaces,

$\beta_{1}=$ class of Banach spaces satisfying (I) with some compact $X$,

$\beta_{2}=$ class of Banach spaces satisfying $\left(A_{2}\right)$,

$\beta_{3}=$ class of Banach spaces satisfying $\left(\mathrm{A}_{1}\right)$,

$\beta_{4}=$ class of Banach spaces satisfying $\left(A_{3}\right)$.

Then we have seen that $\beta=\beta_{1} \supset \beta_{2} \supset \beta_{3} \supset \beta_{4}$. These are all proper inclusions.

Another class is that of all Banach spaces which have the property that all $F_{T}$ are linear. This class properly contains $\beta_{3}$. In addition, it contains all euclidean spaces and all Hilbert (inner product) spaces. The latter spaces are strictly convex, and for strictly convex spaces it can be shown that the linearity of all $F_{T}$ is equivalent to the existence of a Gateaux differential for the norm at every point of the space.

7. The uniqueness theorem and relations between $X$ and $B(X)$. If $B$ is completely regular over a compact $X$, we have seen that the set $M \subset S_{W}^{*}$ of functionals $F_{T}$ is the union of two disjoint closed antipodal 
subsets each homeomorphic to $X$. If there exist two different decompositions of $M$ into a pair of disjoint closed antipodal subsets, say $\Omega,-\Omega$ and $\Omega^{\prime},-\Omega^{\prime}$, it is easy to show that $\Omega$ and $\Omega^{\prime}$ are homeomorphic. Hence a given Banach space can be completely regular over at most one compact space $X[14]$. We say that $B$ determines the topology of $X$. As a special case, if $B=B\left(X_{1}\right)=B\left(X_{2}\right)$ for compact $X_{1}, X_{2}$, then $X_{2}$ is homeomorphic to $X_{1}$ (Banach-Stone theorem) $[3 ; 16]$; "compact" can be replaced by "completely regular and 1st countable" [7].

Thus the problem naturally arises of translating properties of $X$ into properties of $B(X)$ or of subspaces of $B(X)$ completely regular over $X$, and conversely. In this direction, the following theorems can be proved.

If $X$ is completely regular, $B(X)$ is separable if and only if $X$ is compact and metrizable [12].

If $X$ is completely regular, $B(X)$ contains a separable linear subspace completely regular over $X$ if and only if $X$ is separable metric [15].

If $X$ is completely regular, $B(X)$ is reflexive if and only if $X$ consists of a finite number of points [15].

If $X$ is completely regular, $B(X)$ is $n$-dimensional if and only if $X$ consists of $n$ points ( $n$ finite).

$X$ is connected if and only if there does not exist a decomposition of $B(X)$ as a direct sum [7].

A separable metric space $X$ is finite-dimensional if and only if there exists a finite-dimensional closed linear subspace of $B(X)$ which is completely regular over $X$ [15]. If $n$ is the smallest dimension of a euclidean space in which $X$ is homeomorphically imbeddable, there is a closed linear $(n+2)$-dimensional subspace of $B(X)$ containing the constant functions and completely regular over $X$.

This last result indicates that topological properties of a compact finite-dimensional metric space should be translatable into purely metric properties of a finite-dimensional Banach space. For example, let $X$ be a closed subset (not on a circle or line) of the unit disc in the $(x, y)$-plane. Let $B_{X}$ be the set of functions $b=a_{1}\left(x^{2}+y^{2}\right)+a_{2} x$ $+a_{3} y+a_{4}$ on $X$, with $\|b\|=\sup _{x \in X}|b|$. Then $B_{X}$ is a closed linear 4-dimensional subspace of $B(X)$ completely regular over $X$. As $X$ ranges over the subsets of the disc, $B_{X}$ remains unchanged both algebraically and topologically, only its norm varies.

Another result is the following,

A compact space $X$ is a Peano space (compact, locally connected, 
connected metric space) if and only if $B(X)$ is equivalent to a linear subspace $B$ of $B(I)$ ( $I$ = closed interval) with the property that every $T$-set in $B(I)$ intersects $B$ in a $T$-set in $B$.

This is a consequence of the following theorem.

If $X_{1}$ and $X_{2}$ are compact spaces, there exists a continuous mapping of $X_{1}$ onto $X_{2}$ if and only if $B\left(X_{2}\right)$ is equivalent to a linear subspace $B$ of $B\left(X_{1}\right)$ with the property that every $T$-set in $B\left(X_{1}\right)$ intersects $B$ in a $T$-set in $B$.

We prove this as follows. Let $f\left(X_{1}\right)=X_{2}$, where $f$ is continuous. The mapping $F$ of $B\left(X_{2}\right)$ into $B\left(X_{1}\right)$ given by $b_{2} \rightarrow b_{2} f$ for all $b_{2} \in B\left(X_{2}\right)$ is an equivalence between $B\left(X_{2}\right)$ and a closed linear subspace $B$ of $B\left(X_{1}\right)$. A $T$-set in $B\left(X_{1}\right)$ is either of the form $\left\{b_{1} \in B\left(X_{1}\right) \mid b_{1}\left(x_{1}\right)\right.$ $\left.=\left\|b_{1}\right\|\right\}$ or $\left\{b_{1} \in B\left(X_{1}\right) \mid b_{1}\left(x_{1}\right)=-\left\|b_{1}\right\|\right\}$. The intersection of such a $T$ set with $B$ is either $\left\{b_{2} f \in B\left(X_{1}\right) \mid b_{2} f\left(x_{1}\right)=\left\|b_{2}\right\|\right\}$ or $\left\{b_{2} f \in B\left(X_{1}\right) \mid b_{2} f\left(x_{1}\right)\right.$ $\left.=-\left\|b_{2}\right\|\right\}$. These are $T$-sets in $B$ because they are the images under $F$ of the sets $\left\{b_{2} \in B\left(X_{2}\right) \mid b_{2}\left(f\left(x_{1}\right)\right)=\left\|b_{2}\right\|\right\}$ or $\left\{b_{2} \in B\left(X_{2}\right) \mid b_{2}\left(f\left(x_{1}\right)\right)=\right.$ $\left.-\left\|b_{2}\right\|\right\}$ which are $T$-sets in $B\left(X_{2}\right)$. Conversely, let $F$ be an equivalence between $B\left(X_{2}\right)$ and a linear subspace $B$ of $B\left(X_{1}\right)$ with the property that every $T$-set in $B\left(X_{1}\right)$ intersects $B$ in a $T$-set in $B$. The mapping $H$ which takes each $T$-set in $B\left(X_{1}\right)$ into its intersection with $B$ is a mapping onto the set of $T$-sets in $B$ since each $T$-set in $B$ (by Zorn's Lemma) is extendable to one in $B\left(X_{1}\right)$. Let $e=F\left(e_{2}\right)$, where $e_{2} \in B\left(X_{2}\right)$ is the function identically 1 over $X_{2}$. Let $\Omega$ be the set of functionals $F_{T} \in B^{*}$ corresponding to the set $K$ of $T$-sets in $B$ which contain $e$, and let $\Omega_{1}$ be the set of functionals $F_{T}$ in $B^{*}\left(X_{1}\right)$ corresponding to the set of $T$-sets $H^{-1}(K)$. Then $H$ induces a mapping $h\left(\Omega_{1}\right)=\Omega$. If $T_{1}$ is a $T$-set in $B\left(X_{1}\right)$ intersecting $B$ in $T, F_{T_{1}}(b)=F_{T}(b)=\|b\|$ for all $b \in T$; since $F_{T_{1}}$ and $F_{T}$ are both linear functionals over $B$ of norm 1 , they are identical over $B$. From the definition of the weak-* topology, it follows that $h$ is continuous. But $\Omega$ is homeomorphic to $X_{2}$, and $\Omega_{1}$ is homeomorphic to $X_{1}$. Thus $h$ induces a continuous mapping of $X_{1}$ onto $X_{2}$.

8. A theorem on extension of linear functionals. We give now a proof of the following result, a special case of which was used in $\S 3$.

Let $L$ be a linear substance of a Banach space $B$, and let $\alpha$ be a linear functional over $L$ of norm $A$. Then there is a unique normpreserving linear extension $f$ of $\alpha$ over $B$ if and only if the functional $F(b)=\inf _{l \in L}[A\|b+l\|-\alpha(l)]$ is linear, in which case $f=F$ over $B$.

Proof. Over $L, \alpha=F$. For the expression $A\|b+l\|-\alpha(l)$ takes on the value $\alpha(b)$ when $l=-b$, and for a fixed $b \in L$ this is its minimum value since $A\|b+l\|-\alpha(l) \geqq \alpha(b+l)-\alpha(l)=\alpha(b)$.

Now 


$$
\begin{aligned}
-F(-b) & =-\inf _{l \in L}[A\|-b+l\|-\alpha(l)] \\
& =-\inf _{l \in L}[A\|-b-l\|+\alpha(l)] \\
& =\sup _{l \in L}[-A\|-b-l\|-\alpha(l)] .
\end{aligned}
$$

Referring to Banach's proof [2] of the Hahn-Banach theorem, we see that if $b$ is outside $L$ every linear extension $f$ of $\alpha$ of norm $A$ must have the property $-F(-b) \leqq f(b) \leqq F(b)$, and also for each $r$ satisfying $-F(-b) \leqq r \leqq F(b)$ there is a linear extension $f$ of $\alpha$ with norm $A$ and with $f(b)=r$. It follows that if $F$ is linear, $f$ is unique and equals $F$, and conversely if $f$ is unique, $f=F$.

\section{BIBLIOGRAPHY}

1. L. Alaoglu, Weak topologies of normed linear spaces, Ann. of Math. vol. 41 (1940) pp. 252-267.

2. R. F. Arens and J. L. Kelley, Characterizations of the space of continuous functions over a compact Hausdorff space, Trans. Amer. Math. Soc. vol. 62 (1947) pp. 499508.

3. S. Banach, Théorie des operations lineaires, Warsaw, 1932.

4. N. Bourbaki, C. R. Acad. Sci. Paris vol. 206 (1938) pp. 1701-1704.

5. E. Cech, On bicompact spaces, Ann. of Math. vol. 38 (1937) pp. 823-844.

6. J. A. Clarkson, $A$ characterization of $C$-spaces, Ann. of Math. vol. 48 (1947) pp. 845-850.

7. S. Eilenberg, Banach space methods in topology, Ann. of Math. vol. 43 (1942) pp. 568-579.

8. I. Gelfand, Normierte Ringe, Rec. Math. (Mat. Sbornik) N.S. vol. 9 (1941) pp. $3-24$.

9. E. Hewitt, On rings of continuous real-valued functions, Trans. Amer. Math. Soc. vol. 64 (1948) pp. 45-99.

10. S. Kakutani, Concrete representations of abstract (M)-spaces, Ann. of Math. vol. 42 (1941) pp. 994-1024.

11. I. Kaplansky, Topological rings, Bull. Amer. Math. Soc. vol. 54 (1948) pp. 809-826.

12. M. and S. Krein, On an inner characteristic of the set of all continuous functions defined on a bicompact Hausdorff space, C. R. (Doklady) Acad. Sci. URSS vol. 27 (1940) pp. 427-430.

13. D. Milman, Isometry and extremal points, Doklady Academii Nauk SSSR. N.S. vol. 59 (1948) pp. 1241-1244.

14. S. B. Myers, Banach spaces of continuous functions, Ann. of Math. vol. 49 (1948) pp. 132-140.

15. - Spaces of continuous functions, Bull. Amer. Math. Soc. vol. 55 (1949) pp. $402-407$.

16. M. H. Stone, Applications of the theory of Boolean rings to general topology, Trans. Amer. Math. Soc. vol. 41 (1937) pp. 375-481.

17. A. Tychonoff, Über die topologische Erweiterung von Räumen, Math. Ann. vol. 102 (1930) pp. 544-561.

UNIVERSITY OF MICHIGAN 